\title{
A NOTE ON LIMIT THEOREMS FOR MARKOV BRANCHING PROCESSES ${ }^{1}$
}

HOWARD CONNER

Introduction. Let $\left\{Z_{n}^{\prime}, n \geqq 0\right\}, Z_{0}=1$, denote the random variables in a Galton-Watson process with generator

$$
f(s)=E\left[s^{Z_{1}}\right]=\sum_{k=0}^{\infty} p_{k} s^{k},|s| \leqq 1,
$$

where $p_{k}$ is the probability for a particle in the $n$th generation to produce $k$ particles in the $(n+1)$ th generation, independently of $n$.

Let $\left\{Z_{\imath}^{\prime}, t \geqq 0\right\}, Z_{0}^{\prime}=1$, denote the random variables in a continuousparameter Markov branching process with generator

$$
\varepsilon(s)=b\left[\sum_{k=0}^{\infty} q_{k} s^{k}-s\right], \quad|s| \leqq 1,
$$

where $b \Delta+O(\Delta)$ is the probability for a particle existing at time $t$ to die in the interval $(t, t+\Delta)$, independently of $t$, and each $q_{k}\left(q_{1} \equiv 0\right)$ is the probability for a particle dying at time $\tau$ to produce $k$ particles at time $\tau$, independently of $\tau$.

These assumptions imply that $\left\{Z_{n}, n \geqq 0\right\}$ and $\left\{Z_{t}^{\prime}, t \geqq 0\right\}$ are time homogeneous Markov branching processes.

The purpose of this note is to show how the fundamental limit theorems for continuous-parameter Markov branching processes can be derived using the corresponding theorems for Galton-Watson processes. In a brief summary, each limit theorem is a statement about the limiting properties of certain functions, of the $Z_{n}$ or $Z_{t^{-}}^{\prime}$ processes, whose values are probabilities, generating functions or distribution functions. In a natural way, these values associate to each limit theorem a metric space in which the limiting behavior is studied. Using the metric space as a common reference, it is possible to relate the limiting behavior of a $Z_{t}^{\prime}$-process to that of a $Z_{n}$-process.

This relation is achieved by the following result (first proved by J. F. C. Kingman in the special case $I=(0, \infty),[1$, p. 594-597] $)$ :

(K) Let $\pi$ be a continuous mapping of $[0, \infty)$ into a metric space $X$.

Received by the editors October 20, 1965.

1 This work was jointly sponsored by the National Science Foundation under Grant No. GP-4588 and the Mathematics Research Center, United States Army, Madison, Wisconsin, under Contract No. DA-11-022-ORD-2059. 
Assume that for each $t$ in $(0, \infty) \pi$ has either a right-or left-limit at $t$. Let $I=\{a<t<b\}, 0 \leqq a<b \leqq \infty$. A ssume that for each $\Delta \in I$ the sequence $\pi(n \Delta)$ converges to $x(\Delta) \in X$ as $n \rightarrow \infty$. Then

(i) $x(\Delta)$ does not depend on $\Delta \in I$; i.e., $x(\Delta)=x_{0}$ for all $\Delta \in I$,

(ii) $\pi(t)$ converges to $x_{0}$ as $t \rightarrow \infty$.

Proof. The essential fact in this proof is the following property of real numbers, H. T. Croft [2] and J. F. C. Kingman [1].

(C) Assume $U_{k}, k=1,2,3, \cdots$, are open unbounded subsets and $I$ is an open interval, all in $[0, \infty)$. Then there exists a real number $\Delta_{0} \in I$ such that, for each $U_{k}, n \Delta_{0} \in U_{k}$ for infinitely many positive integers $n$.

Assume $\Delta_{1}, \Delta_{2} \in I$ are such that $x\left(\Delta_{1}\right)=\lim _{n} \pi\left(n \Delta_{1}\right) \neq \lim _{n} \pi\left(n \Delta_{2}\right)$ $=x\left(\Delta_{2}\right)$. Then, the convergence of $\pi(n \Delta)$ and the right- or left-limitproperties of $\pi$ imply the existence of two disjoint neighborhoods $N_{1}$ and $N_{2}$, of $x\left(\Delta_{1}\right)$ and $x\left(\Delta_{2}\right)$ respectively, and two unbounded open subsets $U_{1}, U_{2} \subseteq[0, \infty)$ such that, for $t \in U_{j}, \pi(t) \in N_{i}, i=1,2$. Applying property (C) to $U_{1}, U_{2}$ and $I$, there exists a $\Delta_{0} \in I$ such that $n \Delta_{0}$ is in each of $U_{1}$ and $U_{2}$ for infinitely many values of $n$. This contradicts the convergence of $\pi\left(n \Delta_{0}\right)$ to $x\left(\Delta_{0}\right)$. Therefore for some $x_{0} \in X$ and all $\Delta \in I, \lim _{n \rightarrow \infty} \pi(n \Delta)=x_{0}$.

For the same reasons, the nonconvergence of $\pi(t)$ to $x_{0}$, as $t \rightarrow \infty$, would imply the existence of an open unbounded set $U \subseteq[0, \infty)$ and a neighborhood $N$ of $x_{0}$ such that $\pi(t) \notin N$ for all $t \in U$. Applying (C) to $U$ and $I$ gives the existence of a $\Delta_{0} \in I$ such that $n \Delta_{0} \in U$ for infinitely many values of $n$. However this would contradict the convergence of $\pi\left(n \Delta_{0}\right)$ to $x_{0}$; so that, $\lim _{t \rightarrow \infty} \pi(t)=x_{0}$, proving (K).

In the place of an exhaustive development of this method, we have decided to illustrate its applicability by extending some of the fundamental results for Galton-Watson processes.

Acknowledgment. The author expresses his gratitude to $\mathrm{H}$. Kesten and P. Ney for informing him of the recently improved statements of Theorems 1-3 and for suggesting the extension of the local limit law stated in Theorem 4 . The author also expresses his gratitude to $\mathrm{H}$. Kesten and the referee for their comments and, in particular, for pointing out that the condition in Proposition 1 is necessary as well as sufficient.

Preliminaries. Again we let $f(s),(1)$, and $\varepsilon(s),(2)$, be the respective generators for the $Z_{n}$ and $Z_{t}^{\prime}$-processes. We list for convenient reference the following moment calculations:

$$
E\left[Z_{n}\right]=\rho^{n}
$$


where $\rho=f^{\prime}(1)$.

$$
E\left[Z_{t}^{\prime}\right]=e^{a t}
$$

where $a=\mathcal{E}^{\prime}(1)$.

$$
\begin{aligned}
& \operatorname{Var}\left[Z_{n}\right]= \begin{cases}\operatorname{Var}\left[Z_{1}\right] \frac{\rho^{n}\left(\rho^{n}-1\right)}{\rho^{2}-\rho}, & \rho \neq 1, \\
n \operatorname{Var}\left[Z_{1}\right], & \rho=1,\end{cases} \\
& \operatorname{Var}\left[Z_{t}^{\prime}\right]= \begin{cases}\frac{\mathcal{E}^{\prime \prime}(1)-\mathcal{E}^{\prime}(1)}{\mathcal{E}^{\prime}(1)} e^{a t}\left(e^{a t}-1\right), & a \neq 0, \\
\mathcal{E}^{\prime \prime}(1) t, & a=0 .\end{cases}
\end{aligned}
$$

We also have need for some knowledge about the $Z_{t}$-process which allows us to translate weighted moment conditions on its generator into the same conditions on $Z_{t}$. The following is adequate for the purpose of this note.

Proposition 1. Let $\left\{q_{k}\right\}_{k \neq 1}$ be the infinitesimal branching probabilities, (2), for the $Z_{t}^{\prime}$-process. Then for arbitrary positive integer $\alpha$,

$$
\sum_{k=2}^{\infty}\left(k^{\alpha} \log k\right) q_{k}<\infty \Leftrightarrow \sum_{k=2}^{\infty}\left(k^{\alpha} \log k\right) P\left[Z_{\Delta}^{\prime}=k\right]<\infty, \quad \Delta>0 .
$$

(For a coherence in presentation, we have chosen to postpone giving a proof of this proposition until the Appendix of this note.)

Limit theorems. A general type of limiting behavior for a GaltonWatson process with $\rho>0$, (3), is described by the normalized process

$$
W_{n}=\rho^{-n} Z_{n} \text {. }
$$

Since $\left\{W_{n}, n \geqq 0\right\}$ is a nonnegative martingale with $E\left[W_{n}\right]=1$ $[3$, p. 14], we have always the existence with probability 1 of

$$
\lim _{n \rightarrow \infty} W_{n}=W .
$$

However when $\rho \leqq 1, W$ is identically 0 . Unless further restrictions are placed on $f(s)$, this degeneracy can also occur when $\rho>1$.

A complete description of the case $\rho>1$ is given by the following:

Theorem 1 (B. Stigum and H. Kesten, 1965, to Be Published, [4]). Assume $\rho>1$. Let $W_{n}$ and $W$ be defined by (7) and (8). 

(a) If $\sum_{k \geq 2}(k \log k) p_{k}=\infty, \quad$ then $E[W]=0$.
(b) If $\sum_{k \geq 2}(k \log k) p_{k}<\infty, \quad$ then $E[W]=1$,

and the probability distribution of $W$

(i) either has a continuous density except possibly for a discontinuity at the origin,

(ii) or it is concentrated at one point.

In the continuous parameter process with $a$ as defined in (4),

$$
W_{t}^{\prime}=e^{-a t} Z_{t}^{\prime}, \quad a=b\left[h^{\prime}(1)-1\right],
$$

is also a nonnegative martingale with $E\left[W_{t}^{\prime}\right]=1[3$, p. 108], and

$$
\lim _{t \rightarrow \infty} W_{t}^{\prime}=W^{\prime}
$$

exists with probability 1 . Again there is the possibility of $W^{\prime}$ being degenerate.

Our description of the continuous parameter case with $a>0$ is

Theorem $1^{\prime}$. Assume $a>0$. Let $W_{t}^{\prime}$ and $W^{\prime}$ be defined by (10) and (11).

(a) If $\sum_{k \geq 2}(k \log k) q_{k}=\infty$, then $E\left[W^{\prime}\right]=0$.

(b) If $\sum_{k \geqq 2}(k \log k) q_{k}<\infty$, then $E\left[W^{\prime}\right]=1$

and the probability distribution of $W^{\prime}$

(i) either has a continuous density except possibly for a discontinuity at the origin,

(ii) or it is concentrated at one point.

Proof. It is sufficient to restrict our attention to the family of distribution functions defined by

$$
H(t, u)=P\left[e^{-a t} Z_{t}^{\prime} \leqq u\right]=\sum_{k \leqq \exp (a t) u} P\left[Z_{t}^{\prime}=k\right] \quad(t \geqq 0) .
$$

Let us examine $H(t, u)$ more carefully. Assuming $u$ is a point of continuity for $H(\tau, u)$, there exists a $\delta>0$ such that the interval $\left(e^{a t} u-\delta, e^{a \tau} u+\delta\right)$ contains no in teger since the $Z_{t}^{\prime}$-process is aperiodic. Now consider when $t>\tau(t<\tau$ is similarily handled). Then

$$
\begin{aligned}
H(t, u)-H(\tau, u) \leqq & \sum_{k \leqq \exp (a \tau) u}\left|P\left[Z_{t}^{\prime}=k\right]-P\left[Z_{\tau}^{\prime}=k\right]\right| \\
& +\sum_{\exp (a \tau) u \leqq k \leqq \exp (a t) u} P\left[Z_{t}^{\prime}=k\right] .
\end{aligned}
$$

The first summation in (13) involves a finite number of terms, and therefore, using the continuity of each $P\left[Z_{t}^{\prime}=k\right]$ in $t$, tends to 0 as $t$ 
tends to $\tau$. The second summation in (13) vanishes when $\left|e^{a \ell} u-e^{a \tau} u\right|$ $<\delta$. Therefore for each point $u$ of continuity of $H(t, u)$, the left-hand side of (13) tends to 0 as $t$ tends to $\tau$.

This type of convergence for distribution functions is equivalent to convergence in the metric space $\mathfrak{L}[0 \leqq u<\infty]$ of distribution functions $H$ on $[0, \infty)$ with the Levy metric

$d\left(H_{1}, H_{2}\right)=\inf \left\{\epsilon \mid H_{1}(u-\epsilon)-\epsilon \leqq H_{2}(u) \leqq H_{1}(u+\epsilon)+\epsilon\right\}$ [5, p. 33].

Therefore the mapping $\pi$ of $(0, \infty)$ into $\&[0 \leqq u<\infty]$, defined by

$$
\pi(t)=H(t, u),
$$

is continuous. Consequently, we can use $(\mathrm{K})$ with $X=\mathfrak{L}[0 \leqq u<\infty]$, $\pi$ as defined above and $I=(0, \infty)$ to reduce the problem to a study of the convergence in $\mathfrak{L}[0 \leqq u<\infty]$ as $n \rightarrow \infty$ of the distribution functions, $H(n \Delta, u)$, associated with the $Z_{n \Delta}^{\prime}$-process.

For each $\Delta$ in $I,\left\{Z_{n \Delta}^{\prime} ; n \geqq 0\right\}$ is a Galton-Watson process with generator

$$
f^{(\Delta)}(s)=\sum_{k=0}^{\infty} P\left[Z_{\Delta}^{\prime}=k\right] s^{k}, \quad|s| \leqq 1 .
$$

The moment calculations (3) and (4) show $\rho^{(\Delta)} \equiv E\left[Z_{\Delta}^{\prime}\right]=e^{a \Delta}$; and so, the assumption $a>0$ implies $\rho^{(\Delta)}>1$. Moreover, the distribution functions for the normalized process $\left(\rho^{(\Delta)}\right)^{-n} Z_{n \Delta}^{\prime},(7)$, are precisely $H(n \Delta, u)$. Applying Proposition 1 , we have $\sum_{k=2}^{\infty}(k \log k) q_{k}<\infty$ $\Leftrightarrow E\left[Z_{\Delta}^{\prime} \log Z_{\Delta}^{\prime}\right]<\infty, \Delta>0$.

Therefore, each $Z_{n \Delta}^{\prime}$-process satisfies the conditions of Theorem 1 . Using Theorem 1 we can assert that, for each $\Delta$ in $I, H(n \Delta, u)$ converges as $n \rightarrow \infty$ to a nondegenerate distribution $H^{(\Delta)}(u)$ satisfying either (9) (a) or (b).

Applying $(\mathrm{K})$, we know $H^{(\Delta)}(u)$ does not depend on $\Delta$ and setting $H(u)=H^{(\Delta)}(u)$, we can conclude that $H(t, u)$ converges in the metric of $\mathcal{L}[0 \leqq u<\infty]$ to $H(u)$ as $t \rightarrow \infty$, completing the proof.

Our next result is for $Z_{n}$-processes with $\rho<1$.

Theorem 2 (A. Joffe, 1965, [6]). If $f^{\prime}(1)<1$, the conditional generating functions

$$
g_{n}(s)=\frac{f_{n(s)}-f_{n(0)}}{1-f_{n}(s)}, \quad f_{n}(s)=E\left[s^{Z_{n}}\right],
$$

converge uniformly in any closed region, interior to $\{|s|=1\}$, to a limit $g(s)$ which is analytic for $|s|<1$, continuous at $s=1$ with $g(1)=1$ and is therefore a generating function. In particular 


$$
\lim _{n \rightarrow \infty} P\left[Z_{n}=k \mid Z_{n}>0\right]=\text { coefficient of } s^{k} \text { in } g(s) .
$$

Also, $E\left[Z_{1} \log Z_{1}\right]<\infty$ is a necessary and sufficient condition for $g^{\prime}(1)<\infty$.

(A. Joffe has in his paper a different necessary and sufficient condition for $g^{\prime}(1)<\infty$; however, F. Spitzer has shown the two conditions to be equivalent.)

The convergence properties stated in Theorem 2 show the convergence of $g_{n}$ to $g$ with respect to $\max _{0 \leqq \theta \leqq 2 \pi}\left|g_{n}\left(e^{i \theta}\right)-g\left(e^{i \theta}\right)\right|$. Our corresponding theorem for the continuous parameter process is

THEOREM $2^{\prime}$. If $a=\mathcal{E}^{\prime}(1)<0$, the conditional generating functions

$$
g(t, s)=\frac{F(t, s)-F(t, 0)}{1-F(t, 0)}, \quad 0 \leqq t<\infty,|s| \leqq 1,
$$

where $F(t, s)=E\left[s_{t}^{\prime}\right]$, converge uniformly in any closed region, interior to $\{|s|=1\}$, to a generating function $g(s)$. In particular

$$
\lim _{n \rightarrow \infty} P\left[Z_{t}^{\prime}=k \mid Z_{t}^{\prime}>0\right]=\text { coefficient of } s^{k} \text { in } g(s) \text {. }
$$

Moreover, $\sum_{k=2}^{\infty}(k \log k) q_{k}<\infty$ is a sufficient condition for $g^{\prime}(1)<\infty$.

Proof. The generating functions

$$
F(t, s)=\sum_{k=0}^{\infty} P\left[Z_{t}^{\prime}=k\right] s^{k}, \quad|s| \leqq 1, \imath>0,
$$

can be characterized as the unique solution to

$$
\begin{array}{ll}
F(t, s)=s e^{-b t}+\int_{0}^{t} h[F(t-\tau, s)] b e^{-b \tau} d \tau & \text { and } F(t, 1)=1, \\
& 0 \leqq t<\infty,|s| \leqq 1,
\end{array}
$$

where $h(s)=\varepsilon(s)-s$, which for each $t$ is a generating function in $s$. This characterization is sufficient to show $F(t, s)$ is continuous in $(t, s)$ for $0 \leqq t$ and $|s| \leqq 1$. Letting $G[|s| \leqq 1]$ denote the metric space of all generating functions on $|s| \leqq 1$ with the metric $d\left(g_{1}, g_{2}\right)$ $=\max _{0 \leqq \theta \leqq 2 \pi}\left|g_{1}\left(e^{i \theta}\right)-g_{2}\left(e^{i \theta}\right)\right|$, the mapping $\pi$ of $[0, \infty)$ in to $G$, defined by $\pi(t)=F(t, s)$, is therefore continuous.

For each $\Delta>0$, the random variables $\left\{Z_{n \Delta}^{\prime}, n \geqq 0\right\}$ form a GaltonWatson process with generator $f^{(\Delta)}(s)=F(\Delta, s)$. So we again use (K) with $X=G[|s| \leqq 1], \pi(t)=g(t, s)$ and $I=(0, \infty)$ to reduce the problem to the study of the $Z_{n \Delta}^{\prime}$-process, $\Delta$ in $I$. 
The moment calculations (3) and (4) and the assumption $a<0$ imply that $f^{(\Delta) \prime}(1)=e^{a \Delta}<1$. Therefore Theorem 2 can be applied to show the associated conditional generating functions $\pi(n \Delta)=g_{n}^{(\Delta)}$

$$
\left(g_{n}^{(\Delta)}(s)=\frac{f_{n}(s)^{(\Delta)}-f_{n}(0)^{(\Delta)}}{1-f_{n}^{(\Delta)}(0)}, f_{n}^{(\Delta)}(s)=E\left[s^{Z_{n}^{\prime} \Delta}\right]\right)
$$

converge in $G[|s| \leqq 1]$ to a generating function $g^{(\Delta)}$. Furthermore, $g^{(\Delta) \prime}(1)<\infty$ if and only if $\sum_{k=2}^{\infty}(k \log k) q_{k}<\infty$. This follows from Proposition 1 and the second part of Theorem 2 .

Applying $(\mathrm{K})$ with $X, \pi$ and $I$ as defined above, we can conclude that $g^{(\Delta)}$ does not depend on $\Delta, \Delta$ in $I$. Therefore setting $g(s)=g^{(\Delta)}(s)$, $|s| \leqq 1$, we can assert that $g(t, s)$ converges in the metric of $G[|s| \leqq 1]$ to $g(s)$ as $t \rightarrow \infty$. This establishes Theorem $2^{\prime}$.

Another type of limiting behavior for Galton-Watson processes, with $\rho=1$, is given by

Theorem 3 (H. Kesten, P. Ney, F. Spitzer [7, Corollary 1, p. 7]). If $f^{\prime}(1)=1$ and $f^{\prime \prime}(1)<\infty$, the conditional distribution functions

$$
H_{n}(u) \equiv P\left[\frac{2 Z_{n}}{n f^{\prime \prime}(1)} \leqq u \mid Z_{n}>0\right], \quad 0 \leqq u<\infty,
$$

converge for each value of $u$ to $1-e^{-u}$.

The convergence properties stated in Theorem 3 give the weak convergence of the distributions $H_{n}$ to the exponential distribution $e$; i.e., $\lim _{n \rightarrow \infty} H_{n}(u)=e(u)$ at each point $u$ of continuity of $e$, where $e(u)=0$ for $u<0$ and $e(u)=1-e^{-u}$ for $u \geqq 0$. Our extension of Theorem 3 is

Theorem $3^{\prime}$. If $a=\mathcal{E}^{\prime}(1)=1$ and $\mathcal{E}^{\prime \prime}(1)<\infty$ the conditional distribution functions

$$
H(t, u) \equiv P\left[\frac{2 Z_{t}^{\prime}}{\mathcal{E}^{\prime \prime}(1) t} \leqq u \mid Z_{t}^{\prime}>0\right]
$$

converge weakly to the exponential distribution $e(u)$.

Proof. Consider the distribution functions

$$
H(t, u)=P\left[\frac{2 Z_{t}^{\prime}}{\mathcal{E}^{\prime \prime}(1) t} \leqq u \mid Z_{t}^{\prime}>0\right]=\sum_{k \leqq \frac{3}{\mathcal{E}^{\prime \prime}(1) t u}} P\left[Z_{t}^{\prime}=k \mid Z_{t}^{\prime}>0\right] .
$$

Reasoning similar to that used in the proof of Theorem 1' shows the mapping $\pi$ of $(0, \infty)$ into $\&[0 \leqq u<\infty], \pi(t)=H(t, u)$, is continuous in $t$. 
The moment calculations (3)-(6) and the assumptions $a=0$ and $\mathcal{E}^{\prime \prime}(1)<\infty$ imply that $E\left[Z_{\Delta}^{\prime}\right]=1$ and $\operatorname{Var}\left[Z_{\Delta}^{\prime}\right]=\mathcal{E}^{\prime \prime}(1) \Delta<\infty$; so that for each $\Delta>0$ the Galton-Watson process $\left\{Z_{n \Delta}^{\prime}, n \geqq 0\right\}$ satisfies the conditions of Theorem 3. In particular

$$
H(n \Delta, u)=P\left[\frac{2 Z_{n \Delta}^{\prime}}{n f^{(\Delta)^{\prime \prime}(1)}} \leqq u \mid Z_{n \Delta}^{\prime}>0\right]
$$

where $f^{(\Delta)}(s)$ is the generator of the $Z_{n \Delta}^{\prime}$-process, $\Delta>0$.

Therefore each sequence of conditional distribution functions $\{H(n \Delta, u), n \geqq 0\}, \Delta>0$, converges in the Levy metric to the exponential distribution $e(u)$. Applying $(\mathrm{K})$ with $X=\mathfrak{L}[0 \leqq u<\infty]$, $\pi(t)=H(t, u)$ and $I(0, \infty)$, we can assert that $\pi(t)$ and $H(t, u)$ converges as $t \rightarrow \infty$ in the same sense to $e(u)$, completing the proof for Theorem $3^{\prime}$.

A finer analysis for the case $\rho=1$ is the following:

Theorem 4 (H. Kesten, P. Ney, F. Spitzer [7, Theorem 6, p. 33]). Assume $f^{\prime}(1)=1$ and $\sum_{k=2}^{\infty}\left(k^{2} \log k\right) p_{k}<\infty$. Set $d$ $=$ g.c.d. $\left\{k \mid k \geqq 1, p_{k}>0\right\}$. Let $k(n)$ be an integral-valued function of $n$, $n>0$, with $k(n) / n$ bounded. Then

$$
\lim _{n \rightarrow \infty}\left(\frac{n f^{\prime \prime}(1)}{2}\right)^{2} \exp \left[\frac{2 k(n)}{n f^{\prime \prime}(1)}\right] P\left[Z_{n}=k(n)\right]=d .
$$

Our extension of this result is

TheOREM $4^{\prime}$. Assume $a=0$ and $\sum_{k=2}^{\infty}\left(k^{2} \log k\right) q_{k}<\infty$. Let $k(t)$ be an integral-valued function of $t, t>0$, with $k(t) / t$ bounded as $t \rightarrow \infty$. Then

$$
\lim _{t \rightarrow \infty}\left(\frac{t \mathcal{E}^{\prime \prime}(1)}{2}\right)^{2} \exp \left[\frac{2 k(t)}{t \mathcal{E}^{\prime \prime}(1)}\right] P\left[Z_{t}^{\prime}=k(t)\right]=1 .
$$

(This result was first given by Cistyakov [8] when $\mathcal{E}^{\prime \prime}(1)<\infty$.)

Proof. We define the mapping $\pi$ of $(0, \infty)$ into the metric space $X=[0, \infty)$ by

$$
\pi(t)=\left(\frac{t \varepsilon^{\prime \prime}(1)}{2}\right)^{2} \exp \left[\frac{2 k(t)}{t \varepsilon^{\prime \prime}(1)}\right] P\left[Z_{t}^{\prime}=k(t)\right] .
$$

Our assumption about $k(t)$ and the continuity properties of $P\left[Z_{t}^{\prime}=k(t)\right]$ imply that $\pi(t)$ has either a right- or left-limit at each $t>0$. 
When we consider the family of Galton-Watson processes $\left\{Z_{n \Delta}^{\prime} ; n>0\right\}$ with generators $f^{(\Delta)}(s)=\sum_{k=0}^{\infty} P\left(Z_{\Delta}^{\prime}=k\right) s^{k}$, our moment assumptions and the result of Proposition 1 imply that each of these processes satisfies the conditions of Theorem 4 . Also each $Z_{n \Delta}^{\prime}$-process is aperiodic. (The $Z_{t}^{\prime}$-process is aperiodic.) Since $\pi(n \Delta)$ equals the argument of the left-hand side of (15), we can then apply Theorem 4 (with $d=1$ ) and assert the convergence of $\pi(n \Delta)$ as $n \rightarrow \infty$ to 1 for each $\Delta>0$. An application of (K) with $X=[0, \infty), \pi$ as defined above and $I=(0, \infty)$ is sufficient to determine the convergence asserted in (16).

Appendix. (We shall give only an outline for a proof of Proposition 1.)

Outline of Proof. We first define a sequence $\left\{Y_{t}^{(n)}, t>0\right\}$ $(n=1,2, \cdots)$ of auxiliary processes by

$Y_{t}^{(n)}=\infty$ if at least one $n$th generation particle is born at or before time $t$,

$=Z_{t}^{\prime}$ otherwise.

Setting

$$
\begin{aligned}
& p_{k}^{(n)}(t)=P\left[Y_{t}^{(n)}=k \mid Z_{0}^{\prime}=1\right], \\
& p_{j k}^{(n)}(t)=P\left[Y_{t}^{(n)}=k \mid Z_{0}^{\prime}=j\right]
\end{aligned}
$$

it is easily verified that

$$
p_{k}^{(n+1)}(t)=\sum_{j \geq 2} q_{j} \int_{0}^{t} p_{j k}^{(n)}(t-u) b e^{-b u} d u, \quad(k \geqq 2, t>\vdots 0),
$$

and

$$
p_{k}^{(n)}(t) \leqq p_{k}^{(n+1)}(t) \rightarrow p_{k}(t) \quad \text { as } n \rightarrow \infty,
$$

uniformly on bounded $t$-intervals.

Using (17), we have

$$
\sum_{k \geq 2}\left(k^{\alpha} \log k\right) p_{k}^{(n+1)}(t)=\sum_{j \geq 2} q_{j} \int_{0}^{t} \sum_{k \geq 2}\left(k^{\alpha} \log k\right) p_{j k}^{(n)}(t-u) b e^{-b u} d u .
$$

It is sufficient to show

$$
M^{(n)}(t)=\sum_{k \geq 2}\left(k^{\alpha} \log k\right) p_{k}^{(n)}(t)
$$

is uniformly bounded for all $n$ on bounded $t$-intervals. 
Using the convexity of $x^{\alpha} \log x$ and the fact that $Y_{t}^{(n)}$ given $Z_{0}^{\prime}=j$ is distributed as the sum of $j$ independent and identical copies, $Y_{t}^{(n)}(1), \cdots, Y_{t}^{(n)}(j)$, of $Y_{t}^{(n)}$ given $Z_{0}^{\prime}=1$, we can write

$$
\begin{aligned}
E\left[\left(Y_{t}^{(n)}\right)^{\alpha} \log Y_{t}^{(n)}\right. & \left.\mid Z_{0}^{\prime}=j\right] \\
& \leqq \frac{1}{j} \sum_{i=1}^{j} E\left[\left(j Y_{t}^{(n)}(i)\right)^{\alpha} \log j Y_{t}^{(n)}(i) \mid Z_{0}^{\prime}=1\right] \\
& \leqq \sum_{k \geq 2}(j k)^{\alpha} \log j k p_{k}^{(n)}(t) \\
& \leqq\left(j^{\alpha} \log j\right) \sum_{k \geq 2} k^{\alpha} p_{k}^{(n)}(t)+j^{\alpha} \sum_{k \geq 2}\left(k^{\alpha} \log k\right) p_{k}^{(n)}(t) .
\end{aligned}
$$

Substituting (20) into (19) shows

$$
\begin{aligned}
M^{(n+1)}(t) \leqq & {\left[\sum_{j \geq 2}\left(j^{\alpha} \log j\right) q_{j}\right] \int_{0}^{t}\left[\sum_{k \geq 2} k^{\alpha} p_{k}^{(n)}(t-u)\right] b e^{-b u} d u } \\
& +\left(\sum_{j \geq 2} j^{\alpha} q_{j}\right) \int_{0}^{t}\left[\sum_{k \geq 2}\left(k^{\alpha} \log k\right) p_{k}^{(n)}(t-u)\right] b e^{-b u} d u .
\end{aligned}
$$

Consequently the finiteness of $M^{(n)}(t)$ for all $n$ follows readily by induction on $n$ from the finiteness of $\sum_{k \geq 2}\left(k^{\alpha} \log k\right) q_{k}$.

The same techniques can be used to show $M^{(n)}(t)$ is bounded, uniformly in $n$, on any bounded $t$-interval.

The argument is then completed by using the monotone convergence of $p_{\boldsymbol{k}}^{(n)}(t)$ to $p_{k}(t)$, stated in (18), to show

$$
\sum_{k \geq 2}\left(k^{\alpha} \log k\right) p_{k}(t)=\lim _{n \rightarrow \infty} M^{(n)}(t)<\infty
$$

when $\sum_{k_{z 2}}\left(k^{\alpha} \log k\right) q_{k}<\infty$. To prove the converse, set $\phi(k)=k^{\alpha} \log k$ and let $\tau$ be the ramification time for the initial particle. Then

$$
E\left[\phi\left(\sup _{t \leq \Delta} Z_{t}^{\prime}\right)\right] \geqq E\left[\phi\left(Z_{\tau}^{\prime}\right) ; \tau \leqq \Delta\right]
$$

since $\phi(k)$ is increasing.

Since $P\left(Z_{\tau}^{\prime}=k ; \tau \leqq \Delta\right)=q_{k}(1-\exp (-b \Delta))$, we have

$$
E\left[\phi\left(\sup _{t \leq \Delta} Z_{t}^{\prime}\right)\right] \geqq \sum(k \log k) q_{k}(1-\exp (-b \Delta)) .
$$

It is not difficult to show that

$$
E\left[\phi\left(Z_{\Delta}^{\prime}\right)\right]<\infty \Rightarrow E\left[\phi\left(\sup _{t \leq \Delta} Z_{t}^{\prime}\right)\right] \leqq \infty .
$$


The result follows from (21) and (22).

\section{REFERENCES}

1. J. F. C. Kingman, Continuous-time Markov processes, Proc. London Math. Soc. 13 (1963), 593-604.

2. H. T. Croft, A question of limits, Eureka 20 (1957), 11-13.

3. T. E. Harris, The theory of branching processes, Prentice-Hall, Englewood Cliffs, N. J., 1964.

4. B. Stigum and H. Kesten, A limit theorem for multidimensional Galton-Watson processes, preprint.

5. B. V. Gnedenko and A. N. Kolmogorov, Limit distributions for sums of independent random variables, Addison-Wesley, Reading, Mass., 1954.

6. A. Joffe, On the Galton-Watson process with mean less than one, Ann. Math. Statist. (to appear).

7. H. Kesten, P. Ney and F. Spitzer, The Galton-Watson process with mean one and finite variance, preprint.

8. V. P. Čistyakov, Local limit theorems in the theory of branching random processes, Theory of Probability and Its Applications (translation by SIAM) 2 (1957), 360-374.

UNIVERSITY OF WISCONSIN 